\title{
Iniciativas do poder público para o transporte urbano de carga: perspectivas de impacto no cenário brasileiro
}

\author{
Government measures for urban freight transport: perceptions on \\ Brazilian scenario
}

\begin{abstract}
Yesus Emmanuel Medeiros Vieira[a] [ㄱ, Fábio de Rezende Francisco[a] (ㄱ), Renata Albergaria de Mello Bandeira[a] (ㄱ), Orivalde Soares da Silva Junior[a] (ㄱ, Márcio de Almeida D'Agosto[b] [ㄱ, José Geraldo Vidal Vieira[c] ]
\end{abstract}

\author{
[a] Instituto Militar de Engenharia (IME), Rio de Janeiro, RJ, Brasil \\ [b] Universidade Federal do Rio de Janeiro (UFRJ), Rio de Janeiro, RJ, Brasil \\ [c] Universidade Federal de São Carlos (UFSCar), Sorocaba, SP, Brasil
}

Como citar: Vieira, Y. E. M., Francisco, F. R., Bandeira, R. A. M., Silva Junior, O. S., D’Agosto, M. A., \& Vieira, J. G. V. (2021). Iniciativas do poder público para o transporte urbano de carga: perspectivas de impacto no cenário brasileiro. urbe. Revista Brasileira de Gestão Urbana, 13, e20200034. https://doi.org/10.1590/2175-3369.012.e20200034

\section{Resumo}

Este artigo busca identificar, por meio de uma revisão de literatura, as principais iniciativas relacionadas ao transporte urbano de carga (TUC) que vêm sendo implantadas pelo poder público nas cidades em todo o mundo, levantando os impactos que a aplicação dessas medidas gerou em termos de sustentabilidade. Observou-se, por meio da revisão de literatura, que há uma falta de estudos que quantificam as medidas adotadas pelo setor público para o TUC, em especial nos países em desenvolvimento. Assim, desenvolveu-se uma pesquisa survey, com especialistas sobre o tema, com o objetivo de identificar as iniciativas mais indicadas à mitigação dos impactos negativos gerados pelo TUC no contexto das cidades brasileiras, bem como avaliar as principais dificuldades encontradas à implantação das mesmas. As percepções obtidas, comparadas com os resultados quantificados em cidades de diferentes portes ao redor do mundo, fornecem um guia primário que permite aos gestores públicos considerarem não somente as partes interessadas na gestão do TUC, mas também o reflexo que as dimensões populacionais dos municípios possuem sobre a eficácia das medidas pretendidas, possibilitando a escolha de medidas mais adequadas ao contexto de suas cidades.

Palavras-chave: Transporte urbano de carga. Logística da cidade. Políticas públicas.

\begin{abstract}
This paper seeks to identify, through a Literature Review, the main UFT initiatives implemented by public authorities in cities worldwide, identifying the impacts that the application of such measures has generated in terms of sustainability. Considering the lack of quantitative researches on the impact of the implementation of these UFT initiatives by the public sector identified in the study, especially in the developing countries, a survey was carried out with experts on the subject, aiming to identify the initiatives that are most suitable to mitigate the negative impacts generated by the UFT considering the scenario of Brazilian cities, as well as to evaluate the obstacles found in the
\end{abstract}

YEMV é engenheiro de Fortificação e Construção, mestre em Engenharia de Transportes, e-mail: yesuscad@gmail.com FRF é engenheiro civil, e-mail: ffrancisco@metrorio.com.br

RAMB é engenheira de Fortificação e Construção, doutora em Engenharia de Transportes, e-mail: renatabandeira@ime.eb.br

OSSJ é cientista da Computação, doutor em Engenharia de Produção, e-mail: orivalde@ime.eb.br

MAA é engenheiro mecânico e de automóveis, doutor em Engenharia de Transportes, e-mail: dagosto@pet.coppe.ufrj.br

JGVV é cientista da Computação, doutor em Engenharia de Produção, e-mail: jgvvidal@gmail.com 
implementation of such initiatives. Results obtained, contextualized according to the size of the cities where such measures were implemented, provide a primary guide to a public manager that considers not only the stakeholders in the UFT management but also the population context of the municipality, its capacities, and limitations, aiming to select measures with the best possible match between expected goals and results obtained.

Keywords: Urban freight transport. City logistics. Public policies.

\section{Introdução}

O transporte urbano de carga (TUC) é uma atividade importante para o crescimento econômico das zonas urbanas, pois, além do abastecimento, traz impactos diretos no congestionamento do tráfego, na emissão de gases de efeito estufa (GEE), na poluição atmosférica, visual e sonora e em acidentes de trânsito, afetando a qualidade de vida da população urbana (Kin et al., 2017).

É fato que a maioria das políticas implantadas relacionadas ao TUC se baseava em restrições ao tipo de veículo (peso e tamanho) e à acessibilidade em certas regiões de uma cidade, o que dificulta as operações logísticas de forma eficiente, seja pela necessidade de aumentar a frequência das entregas por veículos menores, seja pela utilização de caminhos alternativos por meio de desvios (Zunder \& Ibáñez, 2004; Vieira \& Fransoo, 2015). Contudo, recentemente, os gestores públicos vêm tentando buscar iniciativas para modificar essa abordagem. Por exemplo, em Lyon, França, a partir de 2010, um experimento organizado entre governo e operadores logísticos, com base em um sistema de reserva de espaços de carga e descarga, permitiu a criação de rotas de entrega mais eficientes. Ainda, em Turim, Itália, um modelo de governança colaborativa voluntária entre governo e iniciativa privada permitiu reduções nas emissões de GEE (Marcucci et al., 2017).

No Brasil, essa ainda não é uma realidade. Segundo Dias et al. (2018), as soluções de logística urbana se concentram em medidas restritivas a veículos de carga, que são copiadas de cidade a cidade. A partir de uma survey com gestores públicos de 70 cidades de médio e grande porte, os autores (2018) concluíram que as cidades brasileiras não estão preparadas para desenvolver um plano eficiente de TUC devido à falta de conscientização/negligência às demandas da logística.

Pouca atenção também foi dada, na literatura, ao processo de seleção das medidas públicas voltadas ao TUC, embora estas já se encontrem consolidadas em várias cidades da Europa (Akgün \& Monios, 2018). Observa-se, na literatura, uma falta de pesquisas que quantifiquem as iniciativas adotadas pelo setor público para o TUC, em especial nos países em desenvolvimento. Há também lacunas relativas à compreensão da influência que o porte das cidades possui na seleção e posterior desempenho dessas medidas.

Nesse contexto, torna-se importante buscar iniciativas que permitam um melhor entendimento sobre a eficácia das medidas implantadas pelo poder público para mitigar os impactos negativos gerados pelo TUC nas cidades. Ao se compreender o contexto onde essas iniciativas podem ser empregadas, suas aplicações práticas e seus resultados, é possível auxiliar os planejadores urbanos na busca de soluções personalizadas, de acordo com as necessidades do TUC nas diferentes cidades.

Portanto, o objetivo deste trabalho é abordar as iniciativas relacionadas ao TUC que vêm sendo implantadas pelo poder público nas cidades em todo o mundo, identificando, por meio de uma revisão de literatura, o impacto que a aplicação de tais medidas gera em termos de sustentabilidade. Essa revisão de literatura teve como foco estudos que trazem resultados quantitativos relacionados à aplicação dessas iniciativas, permitindo assim uma avaliação objetiva de seus impactos. Ainda, visando compreender esse tema no contexto de países em desenvolvimento, desenvolveu-se uma pesquisa survey com profissionais e acadêmicos para quantificar os impactos da aplicação de iniciativas relacionadas ao TUC em algumas cidades brasileiras. As análises resultantes deste artigo buscam contribuir como um guia primário aos pesquisadores que queiram explorar a literatura de forma mais rápida e detalhada, bem como permitir aos gestores públicos compreender o impacto de cada medida previamente ao processo decisório referente à sua implantação.

0 artigo está organizado com a seguinte estrutura: a seção 2 apresenta uma revisão das principais iniciativas implantadas pelo poder público com o objetivo de reduzir o impacto do TUC nas cidades. Na seção 3, apresenta-se a metodologia aplicada para o desenvolvimento da revisão de literatura, sendo analisados os resultados obtidos. A seção 4 apresenta os resultados e análises da survey realizada com profissionais e acadêmicos sobre o impacto 
da aplicação de iniciativas relacionadas ao TUC em algumas cidades brasileiras. Por fim, são apresentadas na seção 5 as conclusões, limitações e novas oportunidades de pesquisa.

Iniciativas voltadas para políticas públicas locais sobre o TUC

As autoridades locais estão cada vez mais interessadas em adotar medidas para mitigar as externalidades do TUC, em especial seu impacto ambiental e na qualidade de vida da população urbana (Cherrett et al., 2012). Logo, essa área de pesquisa vem atraindo a atenção da comunidade acadêmica (Akgün \& Monios, 2018). Entretanto, a maioria das pesquisas sobre o tema se preocupa em identificar as medidas públicas relacionadas ao TUC, sendo poucos os estudos que avaliam quantitativamente os resultados obtidos com a adoção dessas iniciativas nas cidades onde foram implantadas (Comi \& Russo, 2016).

Holguín-Veras (2015) propõe um guia de 25 iniciativas para o aperfeiçoamento do TUC, trazendo em seu cerne aspectos sustentáveis. Como referência para este estudo, tomaram-se como base 16 entre as 25 iniciativas propostas por Holguín-Veras (2015), que de fato podem ser aplicadas por parte da administração pública municipal. Também foram consideradas as iniciativas sugeridas por Muñuzuri et al. (2005), das quais 42 podem ser aplicadas pelo poder público. Essas foram avaliadas em suas similaridades de descrição e comparadas às iniciativas já selecionadas de Holguín-Veras (2015), resultando na adição de sete medidas ao conjunto inicial. Assim, avalia-se, neste artigo, um total de 23 iniciativas relacionadas ao TUC, que foram agrupadas segundo a classificação proposta por Holguín-Veras (2015), conforme apresentado na Tabela 1. Destaca-se que tais iniciativas também foram identificadas e propostas em outras publicações, e, embora com terminologias distintas, apresentam conteúdo e significados práticos equivalentes às abordadas neste artigo. Essas publicações também são apresentadas na Tabela 1.

Tabela 1 - Iniciativas voltadas para políticas públicas locais sobre o TUC identificadas na literatura

\begin{tabular}{|c|c|c|c|c|}
\hline Grupo & Código & Iniciativa & Definição & Autores \\
\hline \multirow[t]{3}{*}{$\begin{array}{l}\text { Engajamento de } \\
\text { stakeholders }\end{array}$} & Eng 1 & $\begin{array}{l}\text { Comitê Consultivo de } \\
\text { Frete (Freight Advisory } \\
\text { Committee = FAC) }\end{array}$ & $\begin{array}{l}\text { Grupo composto por diferentes stakeholders, que } \\
\text { funciona como um fórum para discussão de soluções } \\
\text { inovadoras para problemas do TUC. Deve ser } \\
\text { complementado com esforços de divulgação } \\
\text { direcionados para receber feedback de todos os } \\
\text { segmentos da indústria. } \\
\text { Iniciativa com o objetivo de criar entendimento entre } \\
\text { as autoridades quanto à importância do TUC para as } \\
\text { cidades e seu potencial na melhoria do desempenho } \\
\text { do sistema. }\end{array}$ & Holguín-Veras (2015) \\
\hline & Eng 3 & $\begin{array}{l}\text { Comitê Consultivo } \\
\text { Técnico (Technical } \\
\text { Advisory } \\
\text { Committee = TAC) }\end{array}$ & $\begin{array}{l}\text { Fórum no qual representantes do setor público, nas } \\
\text { várias agências de jurisdição, se reúnem para discutir } \\
\text { a política de gestão do TUC. }\end{array}$ & Holguín-Veras (2015) \\
\hline & Eng 4 & $\begin{array}{l}\text { Criar parceria de } \\
\text { qualidade de frete } \\
\text { (Freight Quality } \\
\text { Partnership = FQP) }\end{array}$ & $\begin{array}{l}\text { Parceria voluntária entre grupos do setor público e } \\
\text { privado para promover a implantação de iniciativas } \\
\text { para mitigação dos impactos negativos do TUC. }\end{array}$ & Holguín-Veras (2015) \\
\hline $\begin{array}{l}\text { Estacionamento } \\
\text { / gestão de } \\
\text { áreas de } \\
\text { carregamento }\end{array}$ & Est 3 & $\begin{array}{l}\text { Códigos de } \\
\text { construção } \\
\text { aprimorados } \\
\text { Restrição de acesso } \\
\text { a zonas de } \\
\text { pedestres } \\
\text { Estacionamento em } \\
\text { fila dupla de curta } \\
\text { duração }\end{array}$ & $\begin{array}{c}\text { Legislação que visa prover soluções para o TUC em } \\
\text { regiões de infraestrutura inadequada, tais como } \\
\text { estacionamentos, áreas de embarque em edifícios de } \\
\text { centros urbanos e estacionamentos à margem de } \\
\text { áreas metropolitanas. } \\
\text { Restrição do acesso de veículos de TUC a áreas de } \\
\text { pedestres a certas condições, como: acesso livre a } \\
\text { áreas de pedestres durante a noite a todos os tipos } \\
\text { de veículos, incluindo aqueles usados no TUC; acesso } \\
\text { a zonas de pedestres restrito a veículos de TUC em } \\
\text { determinadas janelas de tempo; uso de veículos } \\
\text { especiais (por exemplo, elétricos) para acessar zonas } \\
\text { de pedestres durante o horário comercial; } \\
\text { discriminação dos tipos de mercadorias e veículos } \\
\text { que podem acessar na área de pedestres. } \\
\text { Permissão de estacionamento em fila dupla para } \\
\text { operações de entrega de carga, limitado a intervalos } \\
\text { não superiores a } 15 \text { minutos. Não objetiva reduzir o } \\
\text { problema do estacionamento em fila dupla no centro } \\
\text { da cidade, mas normalizar até que outras alternativas } \\
\text { válidas sejam fornecidas. }\end{array}$ & $\begin{array}{l}\text { Holguín-Veras (2015) } \\
\text { Rosini et al. (2005), } \\
\text { Muñuzuri et al. (2005), Van } \\
\text { Duin \& Quak (2007), Dasburg } \\
\text { \& Schoemaker (2006), Russo } \\
\text { \& Comi (2011), Thompson \& } \\
\text { Taniguchi (2014), } \\
\text { Creazza et al. (2014) } \\
\\
\text { Muñuzuri et al. (2005) }\end{array}$ \\
\hline
\end{tabular}


Tabela 1 - Continuação...

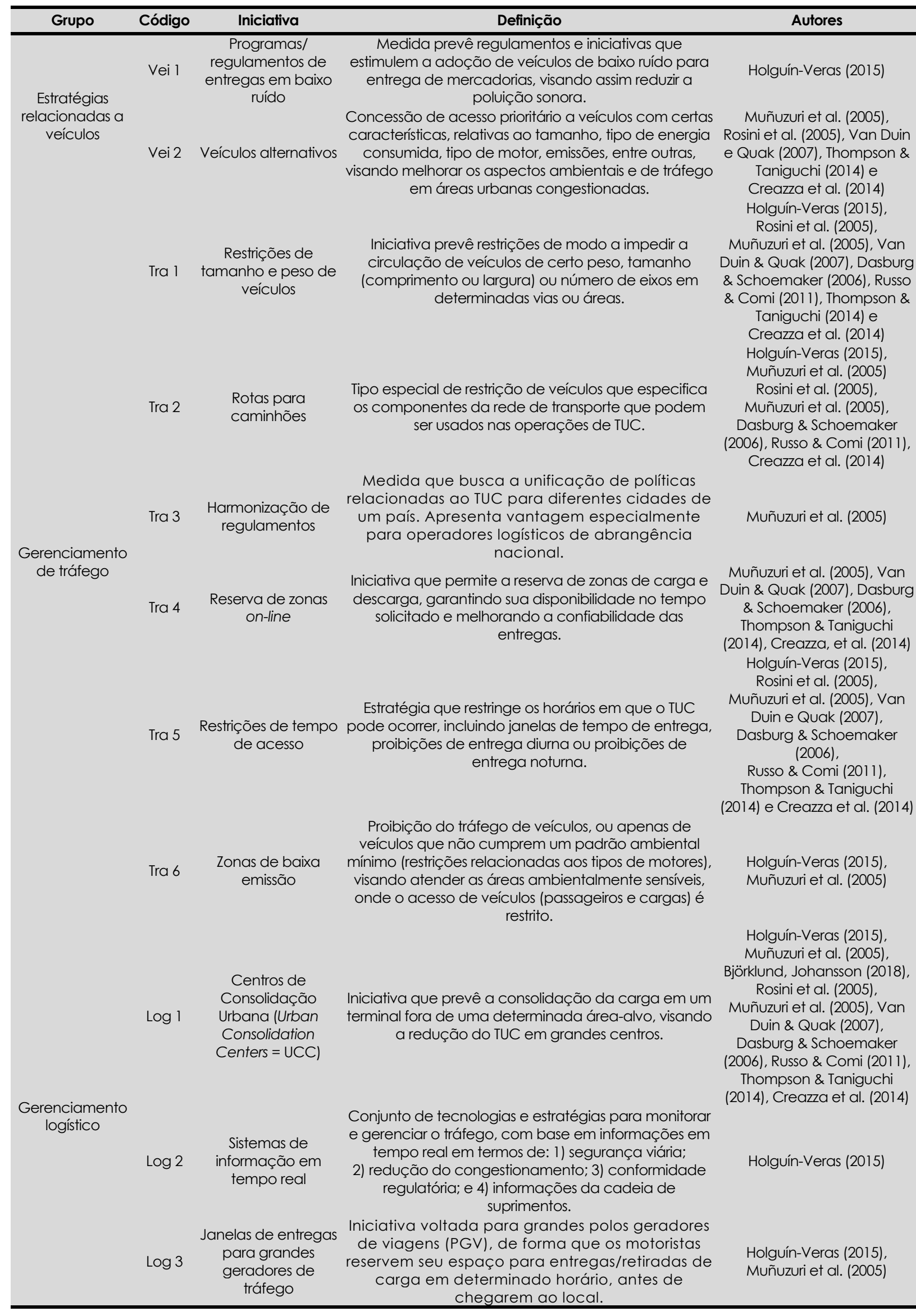


Tabela 1 - Continuação...

\begin{tabular}{|c|c|c|c|c|}
\hline Grupo & Código & Iniciativa & Definição & Autores \\
\hline \multirow{5}{*}{$\begin{array}{l}\text { Preços, } \\
\text { incentivos e } \\
\text { tributação }\end{array}$} & Pre 1 & $\begin{array}{l}\text { Precificação de } \\
\text { estradas }\end{array}$ & $\begin{array}{c}\text { Ferramenta de gestão de demanda em áreas } \\
\text { urbanas para reduzir o tráfego, visando promover } \\
\text { melhor uso da capacidade de transporte e reduzir os } \\
\text { impactos ambientais. Sua aplicação se dá } \\
\text { geralmente pelo uso de pedágios eletrônicos } \\
\text { localizados à margem das cidades em túneis e/ou } \\
\text { pontes, sendo as receitas utilizadas, em geral, para } \\
\text { financiar os custos de construção e manutenção da } \\
\text { infraestrutura urbana. }\end{array}$ & Holguín-Veras (2015) \\
\hline & Pre 2 & $\begin{array}{l}\text { Precificação de } \\
\text { estacionamentos }\end{array}$ & $\begin{array}{c}\text { Medida de cobrança em função do uso de áreas de } \\
\text { estacionamento, podendo se basear em taxas fixas } \\
\text { ou esquemas de precificação variáveis ou } \\
\text { diferenciados. }\end{array}$ & Holguín-Veras (2015) \\
\hline & Pre 3 & $\begin{array}{l}\text { Programas de } \\
\text { certificação / } \\
\text { reconhecimento / } \\
\text { incentivos }\end{array}$ & $\begin{array}{c}\text { Medidas implementadas para incentivar e } \\
\text { recompensar iniciativas sustentáveis em toda a } \\
\text { cadeia de suprimentos. São três os tipos principais: } \\
\text { programas abrangentes, específicos de área e de } \\
\text { concessão. }\end{array}$ & $\begin{array}{l}\text { Holguín-Veras (2015), } \\
\text { Muñuzuri et al. (2005) }\end{array}$ \\
\hline & Pre 4 & Tributação & $\begin{array}{c}\text { Iniciativas usadas para aumentar as receitas e } \\
\text { promover mudanças de comportamento que levem } \\
\text { a benefícios públicos. }\end{array}$ & $\begin{array}{l}\text { Holguín-Veras (2015), } \\
\text { Muñuzuri et al. (2005) }\end{array}$ \\
\hline & Pre 5 & $\begin{array}{l}\text { Acessos } \\
\text { automáticos }\end{array}$ & $\begin{array}{c}\text { Uso de detectores instalados na via, permitindo o } \\
\text { acesso a áreas urbanas de veículos autorizados, } \\
\text { conforme suas características funcionais e/ou } \\
\text { estéticas }\end{array}$ & $\begin{array}{l}\text { Rosini et al. (2005), } \\
\text { Muñuzuri et al. (2005), } \\
\text { Van Duin \& Quak (2007) } \\
\text { Russo \& Comi (2011) e } \\
\text { Creazza et al. (2014) }\end{array}$ \\
\hline
\end{tabular}

\section{Políticas públicas locais voltadas para o TUC}

Para o desenvolvimento dessa revisão de literatura, adotou-se a metodologia proposta por Thomé et al. (2016). A pesquisa foi realizada nas bases de indexações ISI Web of Science e Scopus. Adotou-se a seguinte combinação de palavras-chave: "((urban freight transport*) OR (city logistics)) AND (measure OR policies OR initiative))", com o objetivo de restringir a busca a publicações que realmente se concentrem em aplicações específicas sobre iniciativas voltadas para o TUC e à logística urbana. A busca foi aplicada a títulos, resumos e palavras-chave dos artigos, sendo restrita a artigos publicados em periódicos indexados e revisados por pares nos últimos dez anos (2009 a 2019).

Inicialmente, foram identificadas 82 publicações no Scopus e 127 na ISI Web of Science. Em seguida, foram excluídas as duplicações, resultando em um conjunto de 157 artigos. Foram adotados os seguintes critérios de exclusão no desenvolvimento da revisão de literatura: artigos com conteúdo não relacionado especificamente ao TUC, ou que não contemplam iniciativas públicas relacionadas a esse setor, não foram considerados. Após a revisão dos títulos e resumos, foram mantidos 58 artigos. Foram acrescidos a esses 17 artigos identificados por meio da aplicação do método de snowball (Thomé et al., 2016). Por fim, após a leitura completa, foram excluídos 31 artigos, sendo analisado um total de 44 publicações.

A Figura 1 ilustra a quantidade dessas publicações por ano. Ressalta-se que a ausência de trabalhos identificados no ano de 2009 está alinhada com as observações de Van Duin \& Quak (2007) relativas à existência, naquela época, de uma lacuna real entre as pesquisas e as avaliações práticas sobre as políticas públicas com resultados quantificados. Ainda, a tendência crescente de publicações a partir de 2010 corrobora a premissa adotada neste trabalho de que as pesquisas e iniciativas envolvendo políticas públicas relacionadas ao TUC estão se tornando mais frequentes no meio acadêmico. 


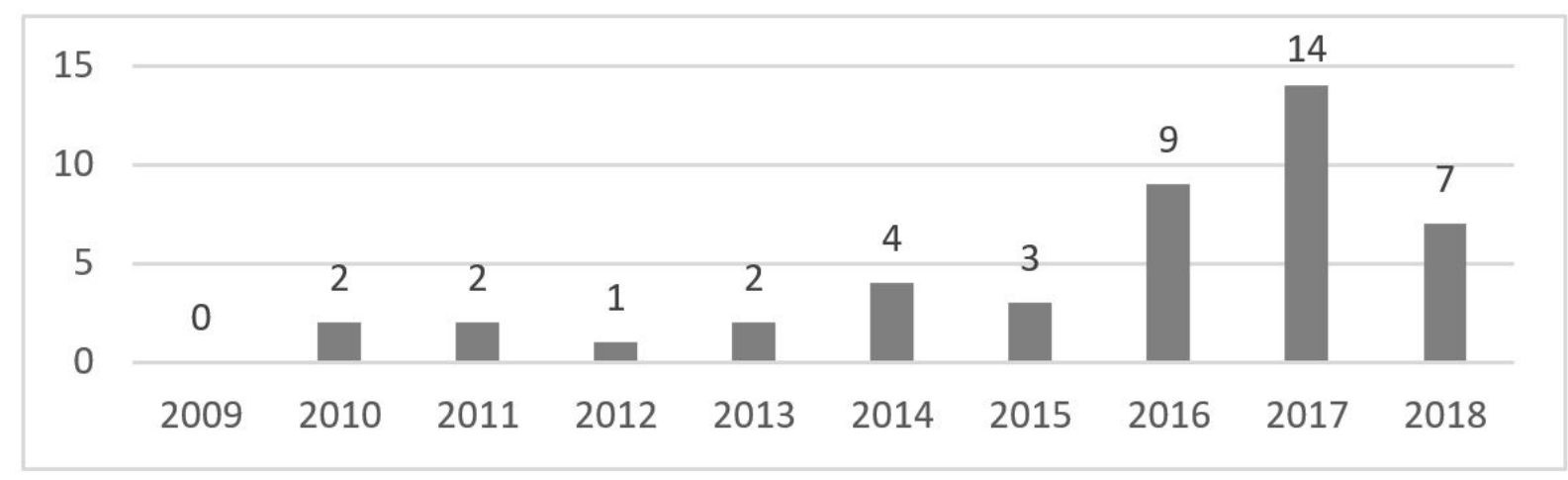

Figura 1 - Quantidade de publicações por ano. Fonte: Elaborado pelos autores.

A revisão de literatura identificou, nos artigos analisados, a descrição de implantação de iniciativas focadas nas políticas públicas relacionadas ao TUC em um total de 49 cidades, sendo a maior parte na Itália (12 cidades), seguida pelos Estados Unidos (7 cidades). Desse total, apenas três são cidades localizadas em países em desenvolvimento. Nesse sentido, Kin et al. (2017) observa que de fato poucas cidades de países em desenvolvimento planejam o TUC, implicando em um arranjo disfuncional de suas logísticas urbanas. Adicionalmente, a maioria das pesquisas relacionadas ao TUC ainda está concentrada em algumas poucas cidades (Dias et al., 2018).

A Figura 2 apresenta a frequência com que cada prática foi discutida no conjunto das publicações analisadas, comparando-as à quantidade de estudos que trouxeram de fato, em termos quantitativos, resultados dos impactos gerados nos centros urbanos pela adoção de políticas públicas relacionadas ao TUC. Observa-se a predominância de abordagens relacionadas às seguintes medidas: iniciativas de janelas de entrega (19 citações), seguidas por Comitê Consultivo de Frete (10 citações) e área de concentração ( 9 citações). Verifica-se assim o padrão dominante de adoção de medidas restritivas, como apontado por Bozzo et al. (2014), que identificou as restrições de acesso como as medidas mais adotadas, ao estudar os 20 maiores projetos de logística urbana implantados nas últimas duas décadas.

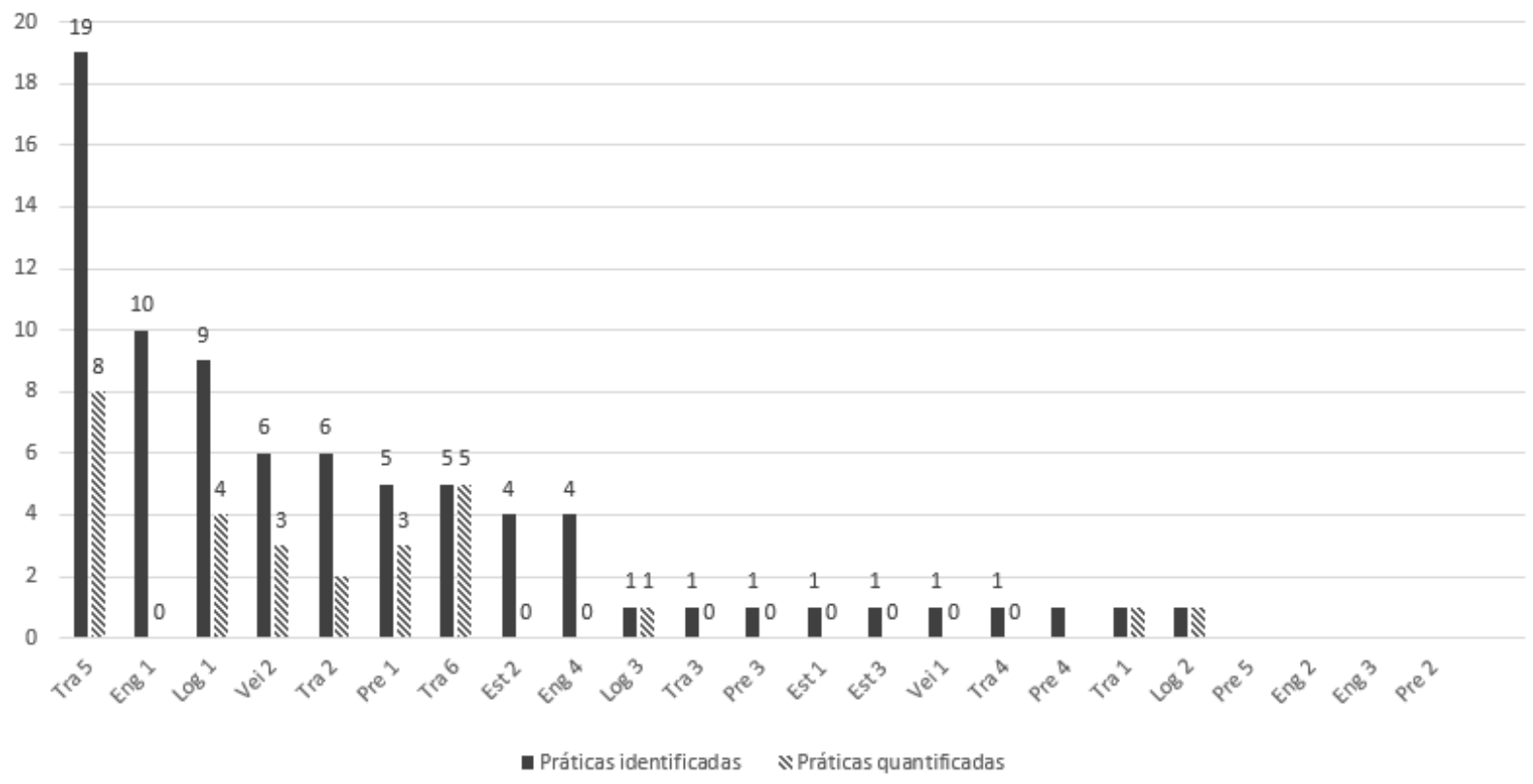

Figura 2 - Comparativo entre iniciativas identificadas e iniciativas quantificadas na revisão de literatura. Fonte: Elaborado pelos autores. 
Nota-se, na Figura 2, que uma reduzida quantidade de estudos realiza análises quantitativas sobre as iniciativas elencadas. Das 23 iniciativas apresentadas na Tabela 1, $19(82,60 \%)$ são identificadas nas publicações selecionadas pela revisão de literatura e apenas $9(39,13 \%)$ dessas apresentaram análises quantitativas. Segundo Russo \& Comi (2011), isso tende a ocorrer devido ao pouco interesse das autoridades locais em validar os programas implantados e devido a dificuldades na obtenção de dados que retratem a realidade do TUC, geralmente atribuídas a pouca colaboração entre as partes envolvidas.

A Figura 3 apresenta a proporção de cidades, segundo o porte, onde as iniciativas foram aplicadas. Adotou-se a classificação do IBGE (2010) para definição do porte das cidades. Observa-se que, das 19 iniciativas identificadas na revisão de literatura, 13 (81\%) são discutidas no contexto de metrópoles, $9(56 \%)$ em cidades de grande porte, 5 (41\%) em cidades de médio porte e $4(33 \%)$ em cidades de pequeno porte, confirmando a necessidade de distintas soluções aos impactos do TUC conforme o aumento das dimensões da cidade estudada.

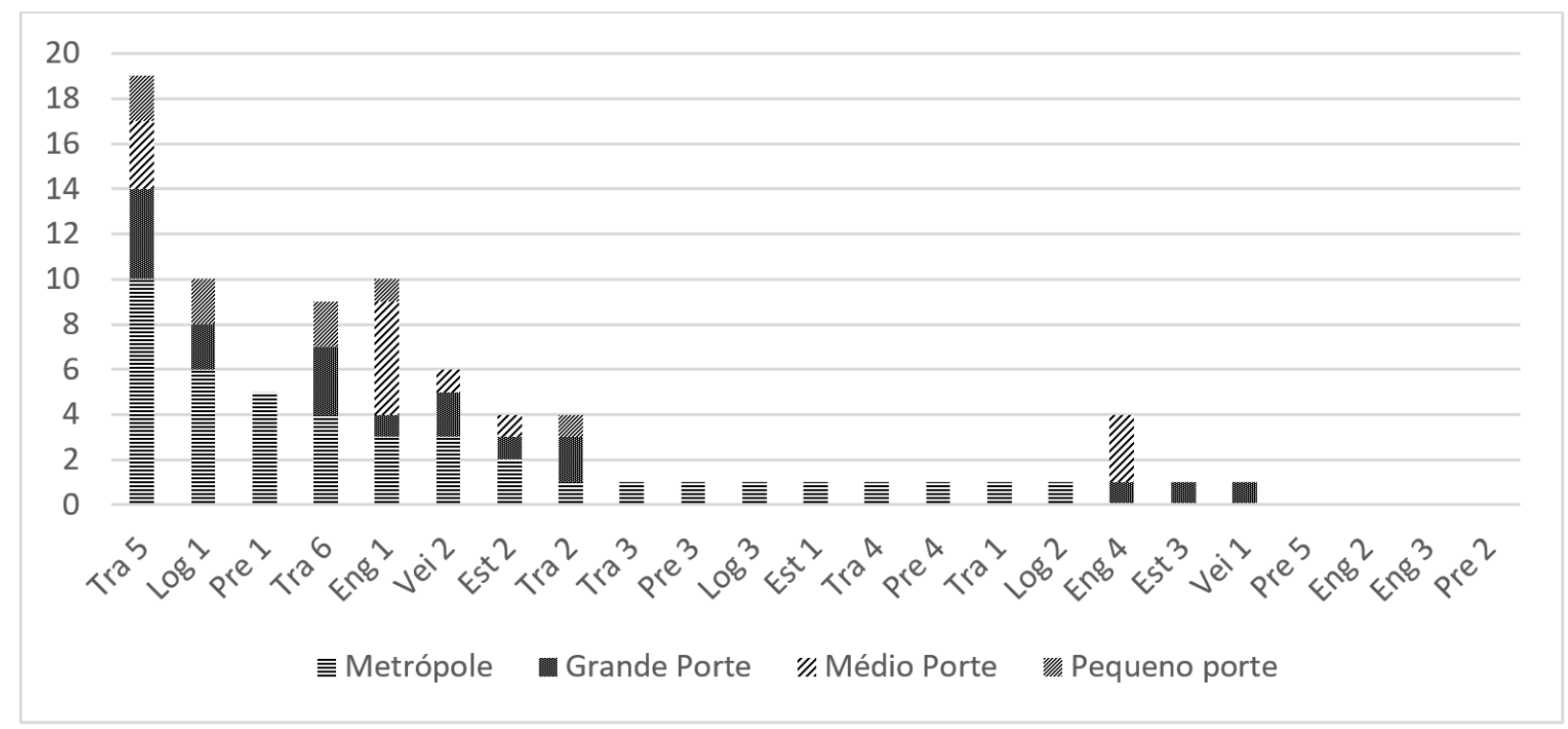

Figura 3 - Distribuição de porte da cidade por prática aplicada.

É possível verificar, por meio da Figura 3, que a iniciativa de restrição de tempo acesso (Tra 5) é abordada sob o viés do gestor público em todos os quatro portes distintos de cidade, sugerindo sua maior abrangência e viabilidade de implantação, independentemente das dimensões urbanas classificadas. Nesse contexto, observa-se que as iniciativas Pre 1, Tra 3, Pre 3, Log 3, Est 1, Tra 4, Pre 4, Tra 1 e Log 2 são relatadas prioritariamente em metrópoles, indicando tratar-se de soluções voltadas a problemas mais característicos de grandes centros urbanos (conurbação urbana, grandes operados de tráfego, necessidade de códigos de construção mais aprimorados).

0 resumo das iniciativas cujos resultados foram de fato quantificados nos artigos é apresentado na Tabela 2, a qual permite verificar que algumas medidas tendem a dar melhores resultados do que outras, conforme o porte da cidade e o objetivo que o gestor público busca atender. 
Tabela 2 - Impactos das iniciativas voltadas a polííicas públicas locais sobre o TUC identificados na literatura

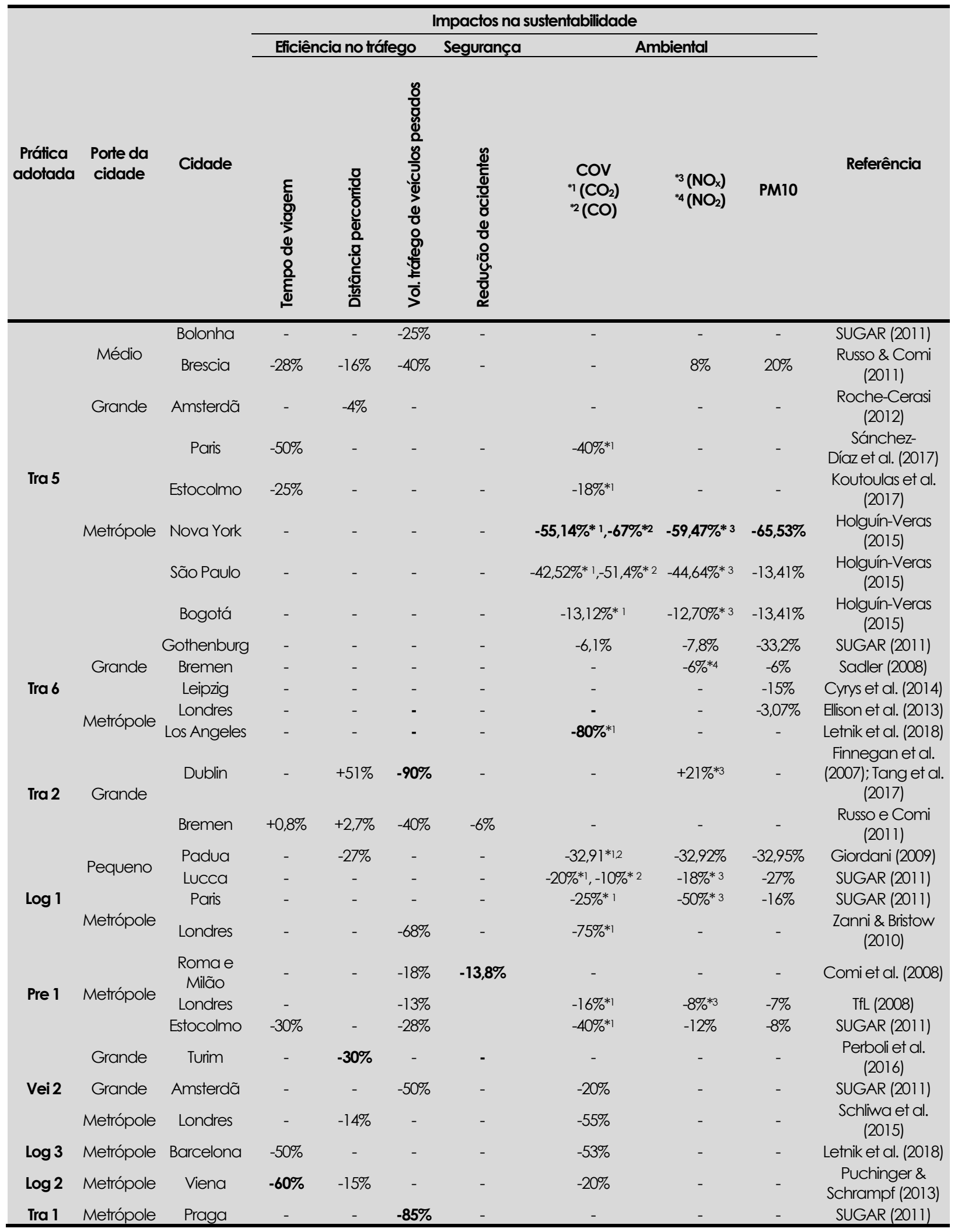

Fonte: Elaborado pelos autores.

Verifica-se que a iniciativa de restrição de tempo acesso (Tra 5) obteve destaque, sob o aspecto da sustentabilidade ambiental, em cidades de médio porte, grande porte e metrópoles. Porém, nas metrópoles, foram obtidos os resultados mais expressivos. De fato, o tráfego menos congestionado em 
metrópoles nos horários de entrega alternativos promove benefícios ambientais e permite deslocamentos com maiores velocidades (Holguín-Veras, 2015). Contudo, a adoção de tal prática pode incorrer em custos adicionais, como pagamento de horas extra e gastos com equipamentos de redução de ruídos. Logo, é necessário verificar se tais investimentos podem ser compensados por uma maior produtividade operacional obtida pelas entregas em horários alternativos, a exemplo do que se verificou em programas piloto conduzidos na Bélgica e no Rio de Janeiro (Verlinde et al., 2010; Silva et al., 2018).

Os impactos das iniciativas de zonas de baixa emissão (Tra 6), assim como a de veículos alternativos (Vei 2), foram quantificados apenas no contexto de cidades de grande porte e nas metrópoles Londres e Los Angeles, tendo a Tra 6 obtido nessa última cidade reduções nas emissões de compostos orgânicos voláteis (COV) até mais expressivas do que a iniciativa Tra 5 alcançou em outras cidades de seu mesmo porte. Em desfavor às iniciativas Tra 6 e Vei 2 pesa, no entanto, a já citada rejeição que tal medida pode encontrar entre os profissionais privados do setor de transporte, por exigir destes investimentos mais elevados com readequação de frota. A viabilidade das mesmas, portanto, pode estar condicionada a cenários específicos mais atrativos a grandes operadores logísticos, a exemplo de zonas portuárias, como ocorreu na metrópole Los Angeles, ou com incentivos do poder público, como o ocorrido em Turim (Letnik et al., 2018; Perboli et al., 2016).

As iniciativas de restrições de tamanho e peso dos veículos (Tra 1) e rotas específicas para os veículos de carga (Tra 2) destacaram-se em termos de melhoria de eficiência do tráfego, obtendo resultados bem semelhantes em escala de magnitude na cidade de grande porte (Dublin) e na metrópole (Praga), já que promoveram a redução do número de veículos de carga circulando em determinados trechos das cidades. Entretanto, para os casos de Dublin e Bremen, ao se considerar a rede de transportes como um todo, a implementação da medida Tra 2 acarretou um aumento em 51\% e 2,7\% respectivamente das distâncias percorridas pelos veículos pesados de carga, elevando em $21 \%$ as emissões de NOx para o caso de Dublin (Russo \& Comi, 2011; Tang et al., 2017). Nesse sentido, o aspecto da eficiência de tráfego é mais bem alcançado pela iniciativa sistema e informação em tempo real (Log 2), a exemplo do identificado na revisão de literatura para a metrópole Viena, com redução de 60\% nas distâncias percorridas (Puchinger \& Schrampf, 2013).

As medidas relativas à criação de áreas de concentração (Log 1) aparecem quantificadas em cidades de pequeno porte e nas metrópoles. No caso das primeiras, a adoção da prática parece estar justificada por suas infraestruturas urbanas menos desenvolvidas, e a consequente necessidade de políticas públicas que reduzam interferências entre os diferentes componentes de mobilidade (caminhões, carros e pedestres), especialmente quando essas cidades possuem centros históricos com vocação turística e são pouco adaptadas ao tráfego de veículos de carga. No caso das metrópoles, a solução visa mitigar os impactos do TUC em centros urbanos já bastante desenvolvidos, apresentando a desvantagem relativa aos altos custos de implantação, conforme destaca Holguín-Veras (2015).

0 entendimento das iniciativas conforme porte da cidade e dos resultados alcançados, expostos na Tabela 2, pode ajudar o gestor público na definição do projeto ou cenário que mais se adeque aos objetivos a serem perseguidos e características de seu município, uma vez que os resultados quantificados para cada prática (magnitude) fornecem algumas indicações primárias do que se pode esperar com cada implantação. Ainda, é importante destacar que determinadas iniciativas costumam ser aplicadas em combinação com outras, de modo a obter melhores resultados.

\section{Aplicação de iniciativas sobre o TUC e seus impactos no cenário brasileiro}

Identificou-se na revisão de literatura a carência de estudos sobre análises quantitativas sobre o impacto da implantação de iniciativas do setor público relacionadas ao TUC, em especial nos países em desenvolvimento. Assim, realizou-se uma survey com o objetivo de identificar as iniciativas mais indicadas para mitigar os impactos negativos gerados pelo TUC nas cidades brasileiras, bem como avaliar os obstáculos encontrados na sua implantação. 
Essa pesquisa utilizou método qualitativo como abordagem de pesquisa, com dados obtidos por meio de entrevistas com especialistas que realizam estudos sobre o TUC ou que possuem experiências práticas com o problema pesquisado, sendo essas então as características da amostra selecionada para a entrevista. A amostra considerada nesse experimento é não probabilística, pois, embora não se possua um conhecimento da população, realiza-se a amostragem com base em perfis já conhecidos da população em estudo: gestores públicos e acadêmicos e profissionais da área privada que atuam sobre o TUC, segmentando a amostra em partes iguais. Dessa maneira, destaca-se que os questionários foram enviados para o maior número possível de pessoas selecionadas, a fim de coletar o maior número de informações possíveis.

Os questionários foram então aplicados a um universo de profissionais da iniciativa privada (23\%), acadêmicos (38\%) e gestores públicos que atuam no sistema de TUC em centros urbanos do Brasil (39\%). As entrevistas foram realizadas em oito municípios: Belo Horizonte-MG, Caicó-RN, Cuiabá-MT, Florianópolis-SC, Natal-RN, Recife-PE, Rio de Janeiro-RJ e Sorocaba-SP. A escolaridade dos entrevistados se distribuiu em: $2^{\circ}$ grau completo (6\%), $3^{\circ}$ grau completo (16\%), pós-graduação $(29 \%)$, mestrado (26\%), doutorado (13\%) e pós-doutorado (10\%). Já o tempo de experiência dos entrevistados seguiu a seguinte distribuição: 0 a 5 anos (36\%); 6 a 10 anos (35\%); 11 a 15 anos (10\%); 16 a 20 anos (13\%), e mais de 20 anos (6\%). Adotou-se no questionário a escala Likert (1 a 5) para avaliar, conforme a perspectiva do entrevistado, o nível de aplicabilidade em determinado município de cada uma das 23 iniciativas listadas na Tabela 1.0 questionário também buscou identificar percepções quanto ao grau de influência de obstáculos à implantação de tais iniciativas, bem como o grau de impacto dos benefícios que seriam decorrentes da aplicação dessas iniciativas nos municípios em questão.

A Figura 4 apresenta o resultado das entrevistas em relação ao nível de aplicabilidade para cada uma das 23 iniciativas identificada na revisão de literatura.

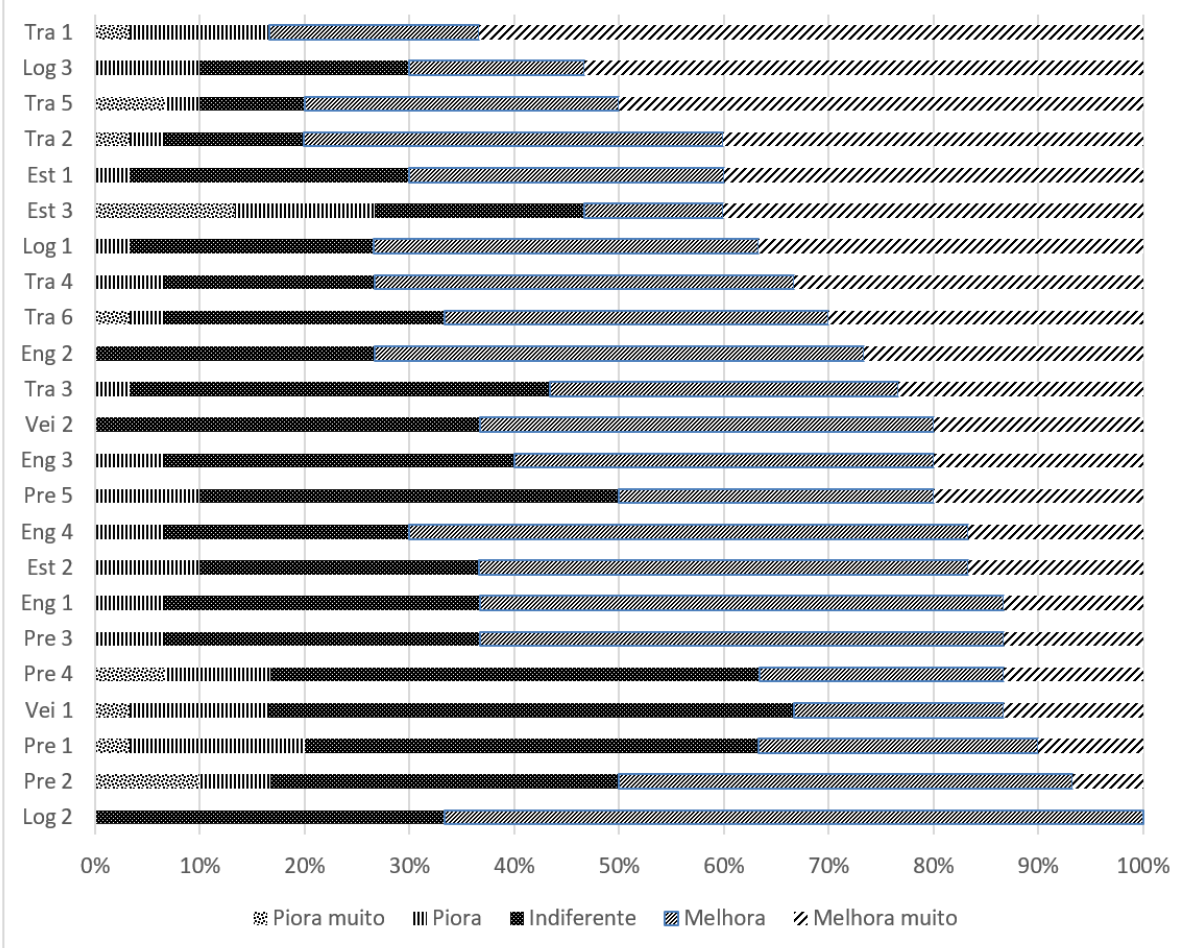

Figura 4 - Aplicabilidade das 23 iniciativas identificadas na RSL no contexto das cidades brasileiras. Fonte: Elaborado pelos autores.

Observa-se, por meio da Figura 4, que as iniciativas restrição de tamanho e peso de veículos (Tra 1), rotas para caminhões (Tra 2) e restrições de tempo de acesso (Tra 5) são as que possuem os maiores 
percentuais acumulados de avalição do tipo "melhora" e "melhora muito". De fato, a predominância de interesses na adoção de medidas restritivas no cenário brasileiro é confirmada em Dias et al. (2018), aspecto que pode ser justificado pelos seus menores custos de implementação (Dablanc, 2007), baixo investimento e baixa manutenção, podendo ser facilmente revogadas se não funcionarem como esperado. Reforçando tal aspecto, verificou-se que essas percepções dos entrevistados encontram semelhanças com os resultados quantitativos obtidos na revisão de literatura (Tabela 2), os quais revelaram que as iniciativas Tra 1 e Tra 2 promoveram maiores impactos na redução de volume de tráfego em metrópoles e cidades de grande porte, destacando-se ainda a iniciativa Tra 5 na redução de emissões de poluentes (NOx, NO2 e PM10), particularmente nas metrópoles. Cabe a ressalva de que, apesar das boas percepções verificadas na survey para as iniciativas Tra 1 e Tra 2, a revisão de literatura permitiu identificar que essas soluções, embora produzam reduções localizadas do volume de tráfego, desencadearam um aumento nas distâncias percorridas e tempos de viagem praticados pelos veículos de carga em cidades de grande porte como Dublin e Bremen, aspectos negativos estes que também foram apontados por Vieira \& Fransoo (2015) na metrópole de São Paulo.

As iniciativas com menores avaliações por parte dos entrevistados foram as relacionadas à precificação de estradas - Pre 1, tributação (Pre 4), precificação de estacionamentos (Pre 2) e restrições de estacionamento duplo de curta duração (Est 3), o que pode estar relacionado à pouca disseminação de pedágios urbanos no Brasil. Nesse quesito, a revisão de literatura identificou (Tabela 2) resultados quantificados para a prática Pre 1 nas metrópoles Roma, Milão e Estocolmo, os quais, em termos de magnitude, foram inferiores aos alcançados por outras medidas, reforçando, sob o aspecto quantitativo, a preterição da mesma. Além disso, observou-se que a rejeição inicial a esse tipo de prática parece ser comum, como verificado em Estocolmo (SUGAR, 2011), onde 75\% dos entrevistados se mostraram contra a implantação do esquema de precificação, tendo essa estatística se revertido em $67 \%$ de aprovação após a consolidação do sistema.

Com a finalidade de confirmar se há variação na percepção de aplicabilidade das iniciativas nas cidades brasileiras de acordo com o local de atuação do respondente ou segundo o porte da cidade, realizaram-se os testes de Kruskal-Wallis e o qui-quadrado para comparação das médias, dado o reduzido tamanho da amostra analisada. Verificou-se que, quanto ao local de atuação do respondente, não houve variação de percepção, ao nível de significância de 5\%, para as 23 iniciativas inseridas no questionário. Porém, com relação ao porte das cidades, foi possível observar, por meio do teste qui-quadrado, diferenças de percepções estatisticamente significantes para as iniciativas Eng 3, Eng 4, Tra 3, Est 3 e Pre 2. Na escala de avaliação de 1 a 5, as médias alcançadas por essas iniciativas nas metrópoles foram de $4,06,4,06,4,25,2,81$ e 2,94, respectivamente, enquanto que nas cidades de grande porte foram de $3,33,3,5,3,16,4,33$ e 3,92, o que revela maior grau de avaliação nas metrópoles para as três primeiras medidas (Eng 3, Eng 4, Tra 3), e nas cidades de grande porte para as duas últimas medidas (Est 3 e Pre 2). De fato, como apontam Dablanc \& Lozano (2013), as metrópoles se caracterizam por possuir alta densidade urbana e espaços mais escassos, sendo por muitas vezes impositiva a operação de carga e descarga com obstrução da via a despeito das restrições impostas, o que pode tornar medidas restritivas de estacionamento menos efetivas em seus ambientes urbanos. Os respondentes também interpretaram que, no contexto das metrópoles brasileiras, de forma semelhante ao observado por Waldhauer et al. (2015) na Cidade do México e no Cairo, as soluções mais efetivas para os problemas de congestionamento em megacidades estão mais relacionadas ao engajamento de stakeholders e gerenciamento do tráfego dentro das cidades.

0 principal benefício esperado nas cidades brasileiras pelos entrevistados diz respeito à diminuição dos congestionamentos, conforme evidenciado na Figura 5. De fato, a proporção de veículos leves de carga (incluindo motocicletas) está aumentando em relação aos veículos pesados para operações TUC. Em São Paulo, aumentos de 158\% para caminhões e 388\% para veículos comerciais leves são esperados entre 2010 e 2040 (Menezes et al., 2017). Essa mudança é parcialmente impulsionada por restrições ao acesso e circulação de caminhões que são implantadas, particularmente, em núcleos urbanos em diferentes cidades do país (Vieira \& Fransoo, 2015). Outros 
benefícios em ordem de importância, conforme elencado pelos entrevistados, são: redução de emissão de GEE e poluentes atmosféricos; aumento de arrecadação de tributos e por último a redução de acidentes de trânsito com veículos de carga.

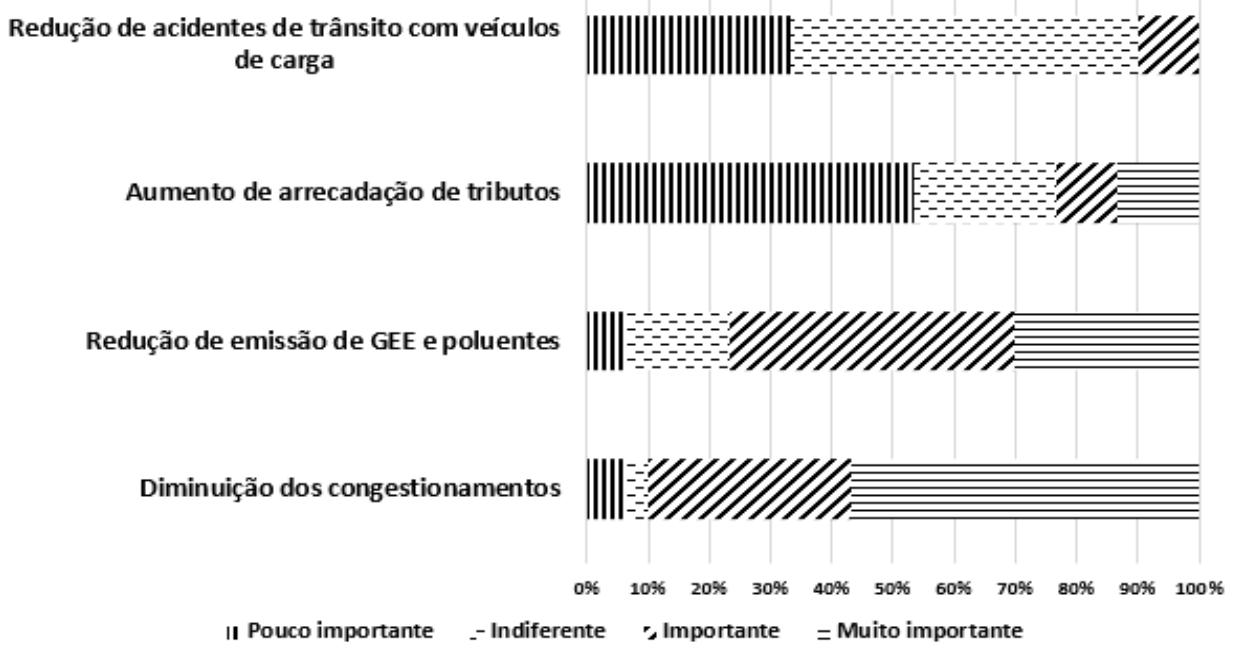

Figura 5 - Principais benefícios da aplicação de iniciativas relacionadas ao TUC. Fonte: Elaborado pelos autores.

Por meio do teste de Kruskal-Wallis e do qui-quadrado, foi possível verificar que a variação na percepção dos respondentes com relação aos benefícios que as iniciativas de TUC podem trazer para as cidades brasileiras ocorre apenas para a redução no número de acidentes de trânsito. Ao nível de significância de $5 \%$, verificou-se a existência de diferenças de percepção entre os profissionais da área privada (média 2,29) e os do setor público (média 1,58), apontando maior preocupação dos particulares com os acidentes envolvendo o frete de carga. Não foram identificadas variações de percepção de benefícios quanto ao porte das cidades.

A Figura 6 apresenta a percepção dos entrevistados com relação aos principais obstáculos para a aplicação das iniciativas relacionadas ao TUC nas cidades brasileiras. Segundo a percepção dos entrevistados, a falta de colaboração dos embarcadores se constitui no principal óbice ao estabelecimento das iniciativas relacionadas ao TUC nas cidades brasileiras, aspecto também observado por Muñuzuri et al. (2012) nas cidades espanholas. Embarcadores e transportadores são relutantes em fornecer informações sobre seus processos logísticos, dificultando a adequada implantação de iniciativas relacionadas ao TUC pelo poder público (Muñuzuri et al., 2012).

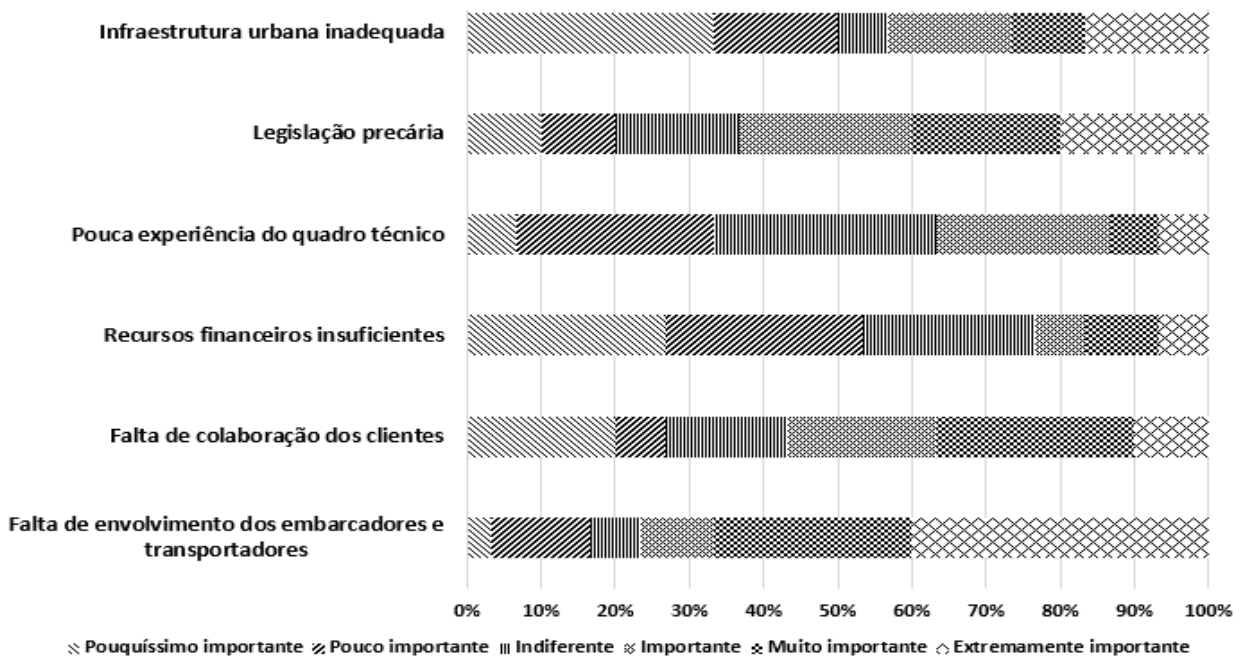

Figura 6 - Principais óbices à aplicação de iniciativas relacionadas ao TUC. Fonte: Elaborado pelos autores. 
Salienta-se que não foram verificadas diferenças de percepção quanto aos óbices citados em relação ao campo de atuação dos respondentes (iniciativa privada, pública ou academia), ao passo que variações com relação ao tamanho da cidade indicaram diferenças de avaliação entre as metrópoles e cidades de grande porte para os óbices de infraestrutura urbana inadequada e legislação precária. 0 grau de percepção desses óbices apresenta tendência de crescimento nas metrópoles em relação às grandes cidades (médias de 4,68 e 4,0 nas metrópoles e 2,75 e 1,58 nas grandes cidades, respectivamente). De fato, a maior fragmentação da demanda em metrópoles, conforme apontam Kin et al. (2017), exige mais da infraestrutura urbana para a circulação de veículos de carga. No entanto, conforme apontado por Dias et al. (2018), a maioria das cidades brasileiras com mais de 500 mil habitantes não possui pessoal treinado para elaboração de um plano de mobilidade urbana de carga, o que pode revelar uma legislação precária ou negligência por parte dos gestores públicos.

\section{Conclusões}

Este artigo realizou, por meio de uma revisão de literatura, a identificação das principais medidas relacionadas ao TUC que vêm sendo implantadas pelo poder público nas cidades em todo o mundo, identificando os impactos que a aplicação de tais iniciativas gerou em termos de sustentabilidade. 0 estudo focou em compreender a influência que o porte das cidades possui na seleção bem como nos resultados quantitativos alcançados por tais medidas, permitindo assim uma avaliação mais concreta e objetiva de seus efeitos nas cidades onde foram implantadas.

A verificação da concordância desses resultados com as percepções do cenário brasileiro se deu por meio de pesquisa realizada com profissionais e acadêmicos da área de transportes, que avaliaram a aplicabilidade das 23 iniciativas identificadas na revisão de literatura e seus impactos, caso fossem implantadas em suas cidades. 0 principal benefício apontado seria a redução de congestionamentos, obtida nas cidades brasileiras principalmente pela aplicação de medidas restritivas de gerenciamento de tráfego. As percepções dos entrevistados, no entanto, enfrentaram algumas ressalvas negativas apontadas pela revisão de literatura. Isso se verificou, por exemplo, com as medidas de restrições de tamanho e peso de veículos e a de rotas para caminhões, as quais, embora tenham reduzido pontualmente o volume de tráfego, comprometeram outros aspectos relacionados à sustentabilidade.

O principal obstáculo apontado pelos respondentes à implantação das iniciativas de TUC foi a falta de envolvimento de embarcadores e transportadores. Embora o foco de pesquisa deste artigo tenha sido em iniciativas promovidas pelas autoridades públicas, diversas ações são resultado de parcerias conjuntas entre autoridades públicas a agentes privados. Assim, embora algumas iniciativas implantadas pelo setor público apresentem efeitos positivos, podem sofrer resistência por parte de outros atores. Logo, é importante reconhecer as preocupações das diferentes partes interessadas, identificando as suas visões sobre o TUC. Tais visões, devidamente ponderadas, permitirão determinar com maior sucesso as políticas ideais para cada cidade.

Por fim, decidir sobre implantação de iniciativas públicas relacionadas ao TUC exige uma avaliação de resultados em termos econômicos, sociais e ambientais. Cada prática deve ser interpretada considerando não apenas os atores e tomadores de decisão envolvidos, mas também levando em conta as características de dimensões populacionais das cidades, uma vez que, sob tais aspectos, as iniciativas referentes ao TUC variaram em aplicabilidade e grau de desempenho, conforme apontou o estudo.

O conjunto dos resultados da revisão de literatura e survey conduzidas neste estudo produz um guia primário aos pesquisadores que queiram explorar a literatura de forma mais detalhada. 0 entendimento dos resultados obtidos neste artigo permite aos gestores públicos um processo de escolha de medidas de gestão do TUC mais criterioso, buscando a melhor adequação entre os objetivos esperados e resultados alcançados, o que é especialmente válido para os contextos de cidades situadas em países em desenvolvimento, carentes desse tipo de estudo. 


\section{Referências}

Akgün, E. Z., \& Monios, J. (2018). Institutional influences on the development of urban freight transport policies by local authorities. In Y. Shiftan, \& M. Kamargianni (Eds.), Preparing for the new era of transport policies: learning from experience (pp. 169-196). Cambridge: Elsevier. http://dx.doi.org/10.1016/bs.atpp.2018.07.004.

Björklund, M., \& Johansson, H. (2018). Urban consolidation centre: a literature review, categorisation, and a future research agenda. International Journal of Physical Distribution \& Logistics Management, 48(8), 745-764. http://dx.doi.org/10.1108/IJPDLM-01-2017-0050.

Bozzo, R., Conca, A., \& Marangon, F. (2014). Decision support system for city logistics: literature review, and guidelines for an ex-ante model. Transportation Research Procedia, 3, 518-527. http://dx.doi.org/10.1016/j.trpro.2014.10.033.

Comi, A., \& Russo, F. (2016). Urban freight transport planning towards green goals: synthetic enviromental evidence from tested results. Sustainability, 8(831), 1-18.

Comi, A., Delle Site, P., Filippi, F., Marcucci, E., \& Nuzzolo, A. (2008). “Differentiated regulation of urban freight traffic: Conceptual framework and examples from Italy. In Proceedings of the 13th International Conference of Hong Kong Society for Transportation Studies. Hong Kong: Hong Kong Society for Transportation Studies.

Creazza, A., Curi, S., \& Dallari, F. (2014). City logistics: panoramica delle best practice nazionali e internazionali (LIUC Papers, No. 271). Castellanza (VA), Italy.

Cyrys, J., Peters, A., Soentgen, J., \& Wichmann, H. (2014). Low emission zones Reduce PM10 mass concentrations and diesel soot in German cities. Journal of the Air \& Waste Management Association, 64(4), 481-487. http://dx.doi.org/10.1080/10962247.2013.868380. PMid:24843918.

Cherrett, T., Allen, J., McLeod, F., Maynard, S., Hickford, A., \& Browne, M. (2012). Understanding urban freight activity - key issues for freight planning. Journal of Transport Geography, 24, 22-32.

http://dx.doi.org/10.1016/j.jtrangeo.2012.05.008.

Dablanc, L. (2007). Goods transport in large European cities: difficult to organize, difficult to modernize. Transportation Research Part A, Policy and Practice, 41(3), 280-285. http://dx.doi.org/10.1016/j.tra.2006.05.005.

Dablanc, L., \& Lozano, A. (2013). Commercial Goods Transport, Mexico City: case study prepared for Global Report on Human Settlements 2013. Recuperado em 30 de janeiro de 2019, de http://unhabitat.org/wpdm-package/ghrs2013-arabic-language-version/2013/

Dasburg, N., \& Schoemaker, J. (2006). D5.2: quantification of urban freight transport effects II: best urban freight solutions II (Report). The Netherlands: Best Urban Freight Solutions.

Dias, J. M., Sobanski, G. B., Silva, J. E. A. R., Oliveira, L. K., \& Vieira, J. G. V. (2018). Are Brazilian cities ready to develop an efficient urban freight mobility plan? urbe. Revista Brasileira de Gestão Urbana, 10(3), 587-599. http://dx.doi.org/10.1590/2175-3369.010.003.ao07.

Ellison, R. B., Greaves, S., \& Hensher, D. A. (2013). Medium term effects of London's low emission zone. In Proceedings of the Transportation Research Board Annual Meeting. Washington.

Finnegan, C., O’Brien, B., \& Traynor, D. (2007) An initial evaluation of Dublin Port Tunnel and the HGV management strategy for the city. In Proceedings of the European Transport Conference. Leiden, The Netherlands.

Giordani, S. (2009). Cityporto Padova: la mobilità sostenibilite delle merci nelle aree urbane. Comune di Padova.

Holguín-Veras, J. (2015). Improving freight system performance in metropolitan area. Washington: TRB's National Cooperative Freight Research Program. Recuperado em 20 de novembro de 2018, de http://pt.slideshare.net/EMBARQNetwork/improving-freight-system performance-in-metropolitan-areas-josholgun-vref-center-of-excellence-for-sustainable-urban-freight-systems-transforming-transportation-2015

Instituto Brasileiro de Geografia e Estatística - IBGE. (2010). Sinopse do censo demográfico de 2010. Recuperado em 30 de janeiro de 2019, de http://www.censo2010.ibge.gov.br/sinopse/index.php?dados=12\&uf= 00 
Kin, B., Verlinde, S., \& Macharis, C. (2017). Sustainable urban freight transport in megacities in emerging markets. Sustainable Cities and Society, 32, 31-41. http://dx.doi.org/10.1016/j.scs.2017.03.011.

Koutoulas, A., Franklin, J. P., \& Eliasson, J. (2017). Assessing nighttime deliveries in Stockholm, Sweden. Transportation Research Record: Journal of the Transportation Research Board, 2605(1), 54-60. https://doi.org/10.3141/2605-05

Letnik, T., Marksel, M., Luppino, G., Bardi, A., \& Božičnik, S. (2018). Review of policies and measures for sustainable and energy efficient urban transport. Energy, 163, 245-257. http://dx.doi.org/10.1016/j.energy.2018.08.096.

Marcucci, E., Gatta, V., Marciani, M., \& Cossu, P. (2017). Measuring the effects of an urban freight policy package defined via a collaborative governance model. Research in Transportation Economics, 65, 3-9.

http://dx.doi.org/10.1016/j.retrec.2017.09.001.

Menezes, E., Maia, A. G., \& Carvalho, C. S. (2017). Effectiveness of low-carbon development strategies: evaluation of policy scenarios for the urban transport sector in a Brazilian megacity. Technological Forecasting and Social Change, 114, 226-241. http://dx.doi.org/10.1016/j.techfore.2016.08.016.

Muñuzuri, J., Cortés, P., Guadix, J., \& Onieva, L. (2012). City logistics in Spain: why it might never work. Cities, 29(2), 133-141. http://dx.doi.org/10.1016/j.cities.2011.03.004.

Muñuzuri, J., Larrañeta, J., Onieva, L., \& Cortés, P. (2005). Solutions applicable by local administrations for urban logistics improvement. Cities, 22(1), 15-28. http://dx.doi.org/10.1016/j.cities.2004.10.003.

Perboli, G., Rosano, M., \& Gobbato, L. (2016) Decision Support system for collaborative freight transortation management: a toll for mixing traditional and green logistics. In Proceedings of the 6th International Conference on Information Systems, Logistics and Supply Chain (ILS 2016). Switzerland: Springer.

Puchinger, J., \& Schrampf, J. (2013). ILOS: intelligent freight logistics in urban areas: freight routing optimisation in Vienna. Vienna: BESTFACT.

Roche-Cerasi, I. (2012). L2.1: state of the art report. Urban logistics practices. Trondheim: SINTEF Teknologi og Samfunn.

Rosini, R., Panebianco, M., \& Zanarini, M. (2005). City Ports project: Interim Report. Recuperado em 4 de fevereiro de 2020, de http://mobilita.regione.emilia-romagna.it/allegati/pubblicazioni/quaderni-servizio pianificazione/quaderno_7.pdf/view

Russo, F., \& Comi, A. (2011). Measures for sustainable freight transportation at urban scale: expected goals and tested results in Europe. Journal of Urban Planning and Development, 137(2), 142-152.

http://dx.doi.org/10.1061/(ASCE)UP.1943-5444.0000052.

Sadler, L. (2008). Detailed assessment London congestion charging. London: Air Quality Office.

Sánchez-Díaz, I., Georén, P., \& Brolinson, M. (2017). Shifting urban freight deliveries to the offpeak hours: a review of theory and practice. Transport Reviews, 37(4), 521-543. http://dx.doi.org/10.1080/01441647.2016.1254691.

Schliwa, G., Armitage, R., Aziz, S., Evans, J., \& Rhoades, J. (2015). Sustainable city logistics - Making cargo cycles viable for urban freight transport. Research in Transportation Business \& Management, 15, 50-57.

http://dx.doi.org/10.1016/j.rtbm.2015.02.001.

Silva, D. M., Bandeira, R. A. M., \& Campos, V. B. G. (2018). Avaliando a sustentabilidade alcançada por meio de entregas noturnas: o caso da distribuição de combustíveis na cidade do Rio de Janeiro. Revista Transportes, 26(2), 54-69. http://dx.doi.org/10.14295/transportes.v26i2.1437.

Sustainable Urban Goods Logistics Achieved by Regional and Local Policies - SUGAR. (2011). City logistics best practices: a handbook for authorities. Bologna.

Tang, J., McNabola, A., Misstear, B., \& Caulfield, B. (2017). An evaluation of the impact of the Dublin Port Tunnel and HGV management strategy on air pollution emissions. Transportation Research Part D, Transport and Environment, 52, 1-14. http://dx.doi.org/10.1016/j.trd.2017.02.009. 
Thomé, M. T., Scavarda, F., \& Scavarda, A. J. (2016). Conducting systematic literature review in operations management. Production Planning and Control, 27(5), 408-420.

http://dx.doi.org/10.1080/09537287.2015.1129464.

Thompson, R. G., \& Taniguchi, E. (2014). Future directions. In E. Taniguchi, \& R. G. Thompson (Eds.), City logistics: mapping the future (Chap. 13, pp. 201-210.). Boca Raton: CRC Press, Taylor \& Francis.

http://dx.doi.org/10.1201/b17715-14.

Transport for London - TfL (2008). Central London congestion charging. impacts monitoring: fifth annual report. London: TfL.

Van Duin, J. H. R., \& Quak, H. J. (2007). City logistics: a chaos between research and policy making? A review. In C. Brebbia (Ed.), Urban transport and the environment in the 21st century (pp. 135-146). Southampton: WitPress. http://dx.doi.org/10.2495/UT070141.

Verlinde, S., Debauche, W., Heemeryck, A., Macharis, C., Van Hoeck, E., \& Witlox, F. (2010). Night-time delivery as a potential option in Belgian urban distribution: a stakeholder approach. In Proceedings of the 12th World Conference on Transport Research Conference. Lisbon, Portugal.

Vieira, J. G. V., \& Fransoo, J. C. (2015). How logistics performance of freight operators is affected by urban freight distribution issues. Transport Policy, 44, 37-47. http://dx.doi.org/10.1016/j.tranpol.2015.06.007.

Waldhauer, N., van der Burgh, M., van der Vorst, J. G. A. J., Bing, X., \& Scheer, F. P. (2015) Perspectives on the distribution of fresh food in emerging metropolises: the cases of Mexico City and Cairo (No. 9789462573642). Wageningen: Food \& Biobased Research. Recuperado em 4 de fevereiro de 2020, de http://edepot.wur.nl/350628

Zanni, A. M., \& Bristow, A. L. (2010). Emissions of CO2 from road freight transport in London: trends and policies for long run reductions. Energy Policy, 38(4), 1774-1786. http://dx.doi.org/10.1016/j.enpol.2009.11.053.

Zunder, T. H., \& Ibáñez, J. N. (2004). Urban freight logistics in the European Union. European Transport, 28, 77-84.

Editor: Fábio Duarte

Recebido: Fev. 04, 2020

Aprovado: Maio 20, 2020 